\title{
A case of retroperitoneal abscess caused by infection of urachal remnant
}

Akira Yoneda ${ }^{1}$, Taiji Hida ${ }^{1}$, Hanako Tetsuo ${ }^{1}$, Saeko Fukui ${ }^{1}$, Shunsuke Murakami ${ }^{1}$, Takayuki Miyoshi $^{1}$, Tatsuya Okamoto ${ }^{1}$, Amane Kitasato ${ }^{1}$, Hiroaki Takeshita ${ }^{1}$, and Tamotsu Kuroki ${ }^{1}$

${ }^{1}$ National Hospital Organisation Nagasaki Medical Center

February 16, 2022

\begin{abstract}
Infection of urachal remnant may cause recurrent abscesses. In the current case report, we describe an urachal remnant infection leading to a retroperitoneal abscess, which is extremely rare condition. In such cases, the recommended treatment is urachal remnant resection.
\end{abstract}

Clinical Image

A case of retroperitoneal abscess caused by infection of urachal remnant

Akira Yoneda, Taiji Hida, Hanako Tetsuo, Saeko Fukui, Shunsuke Murakami, Takayuki Miyoshi, Tatsuya Okamoto, Amane Kitasato, Hiroaki Takeshita, and Tamotsu Kuroki

Department of Surgery, National Hospital Organization Nagasaki Medical Center

Corresponding author: Akira Yoneda, Department of Surgery, National Hospital Organization Nagasaki Medical Center, 2-1001-1 Kubara, Omura, Nagasaki 856-0835, Japan.

Tel.: +81-957-52-3121; Fax: +81-957-54-0292; Email: akira-br@infoseek.jp

Written informed consent was obtained from the patient to publish this report in accordance with the journal's patient consent policy.

\begin{abstract}
Infection of urachal remnant may cause recurrent abscesses. In the current case report, we describe an urachal remnant infection leading to a retroperitoneal abscess, which is extremely rare condition. In such cases, the recommended treatment is urachal remnant resection.
\end{abstract}

Keywords: retroperitoneal abscess, urachal remnant, ureteral resection

\section{Case presentation}

Abscess formation due to infection of urachal remnants is usually localized. A retroperitoneal abscess resulting from such infection is quite rare. [1] [2].

A 65-year-old man with fever and abdominal pain was admitted to our hospital.

Upon computed tomography $(\mathrm{CT})$, we observed gas and extensive abscess formation in the preperitoneal space from the lower abdomen to the pelvis (Fig 1A). We performed emergency laparotomy. Laparoscopic examination of the abdominal cavity revealed no obvious perforation of the gastrointestinal tract and no obvious abscess formation; however, there was marked swelling of the abdominal wall centered on the midline 
umbilical cord, suggesting abscess formation in the same area (Fig 1B). Through a midline incision in the lower abdomen, the Retzius cavity was opened and cleaned, and a drainage tube was placed. On follow-up at 4-6 months postoperatively, a recurrence of retroperitoneal abscess was observed. The patient underwent CT-guided drainage of this new abscess. CT contrast scan showed a slightly hyperdense cord-like structure extending from the umbilical region to the bladder, which was assumed to be a urachal remnant (Fig 1C). This led us to the conclusion that the abscess must have formed in this urachal remnant, subsequently spreading to the retroperitoneum. Hence, we decided to resect this urachal remnant. A laparoscopic ureteral remnant resection was performed. The median cord, i.e., the presumed urachal remnant, was detached from the abdominal wall (Fig 1D).

\section{Acknowledgments}

None.

\section{Conflict of interest}

The authors declare that there is no conflict of interest that could be perceived as prejudicing the impartiality of the research reported herein.

\section{Author contributions}

Akira Yoneda involved in case identification, writing, review and editing the manuscript. Taiji Hida, Hanako Tetsuo, Saeko Fukui, Shunsuke Murakami, Takayuki Miyoshi, Tatsuya Okamoto, Amane Kitasato and Hiroaki Takeshita involved in review, writing, and editing the manuscript. Tamotsu Kuroki involved in supervision of the study.

\section{Ethical Approval}

The study is conducted ethically in accordance with the World Medical Association Declaration of Helsinki.

\section{Consent}

Informed consent for publication and related images has been obtained from the patient.

\section{Data availability statement}

The data that support the findings of this study are available on request from the corresponding author. The data are not publicly due to privacy or ethical restrictions.

\section{References}

1. Sato H, S Furuta, S Tsuji, H KawaseH Kitagawa, The current strategy for urachal remnants. Pediatr Surg Int 2015;31(6):581-587. doi: 10.1007/s00383-015-3712-1

2. Araki M, T Saika, D Araki, Y Kobayashi, S Uehara, T Watanabe, et al. , Laparoscopic management of complicated urachal remnants in adults. World J Urol 2012;30(5):647-650. doi: 10.1007/s00345-012-0829-x.

\section{Figure legends}

Figure 1A: CT. Gas and extensive abscess formation in the preperitoneal space from the lower abdomen to the pelvis.

Figure 1B: Laparoscopic image. There was marked swelling of the abdominal wall centered on the midline umbilical cord, suggesting abscess formation in the same area.

Figure 1C: CT. A slightly hyperdense cord-like structure extending from the umbilical region to the bladder, which was assumed to be a urachal remnant

Figure 1D: Laparoscopic image. The median cord, i.e., the presumed urachal remnant, was detached from the abdominal wall. 
Fig. 1A

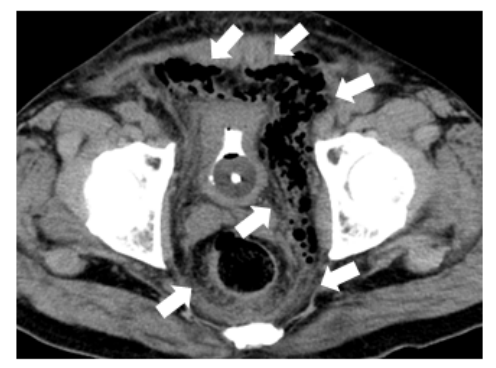

Fig. $1 \mathrm{C}$

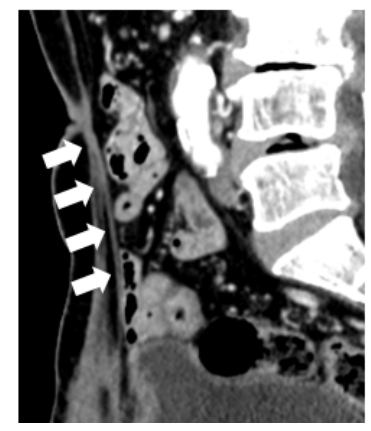

Fig. 1B

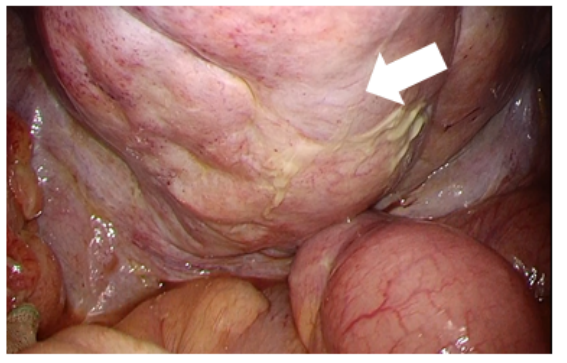

Fig. 1D

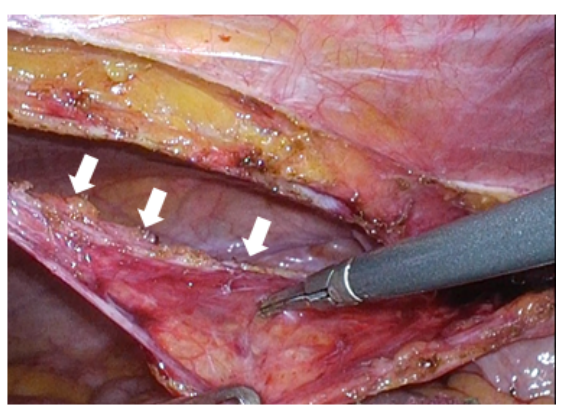

